\title{
Classification of degenerative segment disease in adults with deformity of the lumbar or thoracolumbar spine
}

\author{
Pedro Berjano • Claudio Lamartina
}

Received: 6 July 2013/Revised: 25 January 2014/ Accepted: 25 January 2014/Published online: 23 February 2014

(C) The Author(s) 2014. This article is published with open access at Springerlink.com

\begin{abstract}
Background Lumbar and thoracolumbar deformity in the adult is a condition with impairment of health status that can need surgical treatment. In contrast with adolescent deformity, where magnitude of the curve plays a significant role in surgical indication, the aspects relevant in adult deformity are pain and dysfunction that correlate with segment degeneration and imbalance. Previous classifications of adult deformity have been of little use for surgical planning. Methods Chart review and classification of radiographic and clinical findings. A classification of degenerative disc disease based on distribution of diseased segments and balance status of the spine is presented.

Results Four main categories are presented: Type I (limited nonapical segment disease), Type II (limited apical segment disease), Type III (extended segment disease-apical and nonapical), Type IV (imbalanced spine: IVa, sagittally imbalanced; IVb, sagittally and coronally imbalanced).

Discussion and conclusion Types I and II can be treated by fusion of a selective area of the curve. Type III needs fusion of all the extension of the coronal curve. Type IV usually needs aggressive corrective procedures, frequently including posterior tricolumnar osteotomies. This
\end{abstract}

\footnotetext{
P. Berjano

IVth Spine Surgery Division, IRCCS Istituto Ortopedico

Galeazzi, Milan, Italy

P. Berjano $(\square)$

Via Riccardo Galeazzi 4, Milan, Italy

e-mail: pberjano@gmail.com

C. Lamartina

IInd Spine Surgery Division, IRCCS Istituto Ortopedico

Galeazzi, Milan, Italy
}

classification permits interpreting the extension and magnitude of the disease and can help establish a surgical plan regarding selective fusion and methods of sagittal correction. Future research is needed to validate the classification.

Keywords Classification - Degenerative disc disease . Adult thoracolumbar deformity - Sagittal balance . Adult scoliosis

\section{Introduction}

Deformity in the adult spine can be the consequence of persistence in the adult life of congenital, early-onset or adolescent deformity, or can present clinically as the progression or degeneration of a previously existing curve, a new presentation of deformity due to degeneration of the motion segments of the spine (mainly, but not only, the intervertebral discs) or the result of osteoporotic fractures, tumor or other causes [1].

As opposed to adolescent scoliosis, where pain and disability are not frequently present in association with the deformity, and the degree and type of the deformity and its progression before skeletal maturity is the most relevant aspect of the pathology, in adult patients with spinal deformity (probably with the only exception of young adults with severe deformity in whom a surgical treatment that should have been indicated in adolescence was not performed), pain and disability are the most relevant findings to take the decision of surgical treatment. In these patients, pain can be the consequence of degenerative disc disease, facet degeneration, nerve compression, other soft tissue degeneration, sagittal or coronal (the latter with a lesser impact on pain and disability) imbalance, or a combination of these factors [2-4]. 
Surgical treatment is challenging and causes frequent complications, such as neurological impairment, infection, wound problems, implant-related complications, progression of deformity and death [5, 6].

Several orientations of treatment have been proposed: neural decompression alone, decompression with limited fusion or curve correction with extended fusion to all the curve or curves [7]. The first modality's weakness is the risk of curve decompensation and impairment, leading to the need of revision. The disadvantages of extensive fusion include a higher complication rate and greater loss of spinal range of motion.

Current classifications of adult deformity [1, 8-11], have outlined the etiological aspects of adult deformity and outcome-related morphological aspects, improving our comprehension of the pathology, informing about the cause of the deformity (Aebi's classification) or establishing criteria of severity of the curve (Schwab's classification). One of the limitations of the current classifications is their inability to identify candidates for selective fusion of one area of the curve or to assist the surgeon with surgical planning. In this paper, a new treatment-oriented classification system is presented for degenerative (spinal) segment disease (DSD) in adults with deformity affecting the lumbar and thoracolumbar spine (the most frequent presentation site of adult symptomatic scoliosis).

\section{Methods}

\section{Rationale for a classification}

This classification method has been proposed as guidance to surgical planning. The goals of the classification are to provide the rationale regarding:

- Indication, when feasible, of selective fusions rather than fusions of the whole curve area to reduce surgical risk and maintain as much range of motion as possible of the spine.

- Criteria to reduce the risk of early junctional disease and decompensation of the deformity.

- Selection of appropriate methods to obtain correction of sagittal misalignment.

The most important factors contributing to the presentation of symptoms and the indication of surgery in adult deformity are pain and disability, which are usually secondary to degeneration of the motion segment, progression of the deformity or spinal imbalance, mainly in the sagittal plane. DSD, mostly but not only in the form of disc degeneration, is itself the main cause of presentation of de novo adult scoliosis and in some instances can be the cause of progression of idiopathic scoliosis in the adult age. DSD is also the main etiological factor in loss of lumbar lordosis or increase of thoracic kyphosis, leading to sagittal misalignment and imbalance. DSD is also a frequent source of axial pain in adult scoliosis. Foraminal and canal stenosis due to distortion of the spinal morphology are a consequence of DSD too [7].

Surgical planning for DSD in patients with spinal deformity differs from planning in DSD patients without deformity. In the former case, the choice of fusion levels depends on the relationship or the diseased discs to certain areas of the curve. Specially, a fusion area ending just near the apical region of a scoliotic curve is a known factor for increased risk of adjacent disc disease and decompensation, and for this reason limited fusion ending at one side of the apical area of the scoliotic curve should be avoided. In these cases, ending the fusion far from the apical area or, when it is not possible, extending fusion across the apex should always be preferred [12].

On the other hand, sagittal imbalance has demonstrated to be an independent determinant of outcome in adult deformity [3, 4]. Its correction is a main predictor of clinical success in surgery for adult spine deformity [5, 13]. Furthermore, being the correction of sagittal imbalance one of the main targets of this surgery, the amount of necessary sagittal correction significantly determines the choice of surgical strategy. Standard surgical methods applied to degenerative disc disease (intraoperative patient positioning, use of appropriate interbody implants, rod contouring, segmental compression) can frequently obtain an increase of as much as $25^{\circ}$ in the sagittal plane, depending on the number of levels to be treated and the rigidity of the spine [21]. Some of these methods of correction can be applied with minimally invasive surgical techniques. Greater improvement of lumbar lordosis, though in some cases possible with these standard methods, can more predictably be achieved with more "powerful" correction methods [14]. Currently, multilevel Smith-Petersen osteotomies (SPO), and to a greater extent pedicle subtraction osteotomy (PSO), have been the most effective methods to obtain an increase of lordosis in the range of $25^{\circ}-40^{\circ}$. These techniques differ from standard surgical techniques for degenerative spine disease in their greater technical difficulty and increased risk.

The classification of the deformity is mainly based on standing full-spine frontal and lateral radiographs, supineextension lateral radiographs of the spine and MRI or CT scan. Clinical examination and additional testing as selective blocks or provocative discography and supine frontal side-bending films can be required in some patients for complete classification and surgical planning.

This classification has been conceived to provide a rationale for surgical planning in adults with thoracolumbar or lumbar deformities and degeneration of the segments of 
motion in some part of the involved area of the spine. These patients mainly correspond to the "L" (thoracolumbar/lumbar only) type in the SRS-Schwab's classification or to the " $\mathrm{N}$ " (no major coronal deformity) type with main involvement of the thoracolumbar or lumbar spine [11]. Though the concepts included in this classification can be applied to some cases of idiopathic deformity in the adult (those cases with smaller coronal curves and degenerated motion segments that make the curve progress and/or become symptomatic), this classification system has its main application in patients with de novo scoliosis [1]; the extension of these principles to severe, progressive idiopathic scoliosis in the adult is not suggested by the authors.

\section{Study design}

Chart review and classification of radiographic and clinical findings.

\section{Classification principles}

The classification of degenerative disc disease has been based on two criteria: the distribution of degenerated symptomatic motion segments respect to distinct areas of the main coronal curve (the apical and/or the end area) and the spinal balance of the patient.

The apical area of the curve is defined as the three apical discs (when the apex of the main curve is a disc space) or the two discs around the apical vertebra, one above and one below (when the apex of the main curve is a vertebra). The end area (nonapical area) is defined as the discs near the end vertebrae of the main lumbar or thoracolumbar curve.

Sagittal imbalance is defined [12] as anterior displacement of the $\mathrm{C} 7$ plumbline (more than $5 \mathrm{~cm}$ in front of the posterior corner of the S1 endplate) or the presence of increased pelvic tilt (more than the theoretical pelvic tilt according to Vialle's formula [15]). Correction of the sagittal misalignment (sagittal imbalance) has been demonstrated to be a main predictor of the clinical outcomes and revision rate $[4,13,16]$. Several methods have been described to calculate the amount of necessary correction in the sagittal plane to normalize the spinal sagittal alignment [17-20]. In the present classification, sagittal imbalance is classified as mild (when $<25$ extra degrees of lordosis are needed to restore sagittal alignment) or severe (when an increase of more than $25^{\circ}$ of lordosis is needed). The rationale for this distinction is that increases of lordosis of more that $25^{\circ}$ cannot reliably be obtained with standard procedures, such as interbody cage placement or rod contouring, and usually require aggressive procedures such as pedicle subtraction osteotomies to obtain correction [21], with a substantial impact on surgical strategy.
Table 1 Classification of degenerative segment disease in adults with lumbar or thoracolumbar deformity

\begin{tabular}{|c|c|c|}
\hline $\begin{array}{l}\text { Type I: localized } \\
\text { nonapical DSD } \\
\text { (balanced) }\end{array}$ & $\begin{array}{l}\text { Symptomatic DSD is } \\
\text { present at nonapical } \\
\text { segments of the main } \\
\text { coronal curve }\end{array}$ & $\begin{array}{l}\text { The deformity does not } \\
\text { affect the surgical } \\
\text { plan. Selective } \\
\text { fusion can be } \\
\text { performed, at the same } \\
\text { levels that would be } \\
\text { done in simple } \\
\text { degenerative disease. } \\
\text { Decompression } \\
\text { without fusion in } \\
\text { selected patients can } \\
\text { be an alternative. In } \\
\text { some cases, fusion } \\
\text { should be extended to } \\
\text { the sacrum (see text) }\end{array}$ \\
\hline $\begin{array}{l}\text { Type II: localized } \\
\text { apical DSD } \\
\text { (balanced) }\end{array}$ & $\begin{array}{l}\text { Symptomatic DSD is } \\
\text { present at the apical } \\
\text { segments of the main } \\
\text { coronal curve }\end{array}$ & $\begin{array}{l}\text { Selective fusion is } \\
\text { possible, but it should } \\
\text { extend at both sides of } \\
\text { the apex of the curve. } \\
\text { An exception is } \\
\text { single-level DSD with } \\
\text { the degenerated disc } \\
\text { being at the apex of } \\
\text { the coronal curve. } \\
\text { Correction of the } \\
\text { curve with short } \\
\text { fusion permits } \\
\text { restoring the } \\
\text { alignment of adjacent } \\
\text { flexible discs }\end{array}$ \\
\hline $\begin{array}{l}\text { Type III: extensive } \\
\text { (apical and } \\
\text { nonapical) DSD } \\
\text { (balanced) }\end{array}$ & $\begin{array}{l}\text { Symptomatic DSD is } \\
\text { present all over the } \\
\text { main curve or beyond } \\
\text { (frequently into the } \\
\text { lumbosacral junction) }\end{array}$ & $\begin{array}{l}\text { Fusion should include } \\
\text { all the main curve and } \\
\text { extend if needed to the } \\
\text { lumbosacral junction }\end{array}$ \\
\hline $\begin{array}{l}\text { Type IV: } \\
\text { imbalanced spine }\end{array}$ & $\begin{array}{l}\text { Loss of lordosis is } \\
>25^{\circ} \text { and the sagittal } \\
\text { deformity does not } \\
\text { correct in extension } \\
\text { films }\end{array}$ & $\begin{array}{l}\text { Powerful correction } \\
\text { methods (tricolumnar } \\
\text { osteotomies or } \\
\text { aggressive release) are } \\
\text { needed }\end{array}$ \\
\hline $\begin{array}{l}\text { Type IVa: sagitally } \\
\text { imbalanced }\end{array}$ & $\begin{array}{l}\text { Severe sagittal } \\
\text { imbalance with no or } \\
\text { slight coronal } \\
\text { imbalance }\end{array}$ & \\
\hline $\begin{array}{l}\text { Type IVb: } \\
\text { coronally and } \\
\text { sagitally } \\
\text { imbalanced }\end{array}$ & $\begin{array}{l}\text { Severe imbalance both } \\
\text { in the coronal and } \\
\text { sagittal planes }\end{array}$ & \\
\hline
\end{tabular}

As some patients presenting in standing radiographs with severe sagittal imbalance but having a flexible spine (those with the so-called "collapsed spine") can be completely corrected by positioning, we suggest that patients be evaluated with lateral films in extension-supine position, with the use of a bolster; subjects with complete or almost complete correction of the sagittal deformity under this manoeuver should be considered to have mild sagittal 

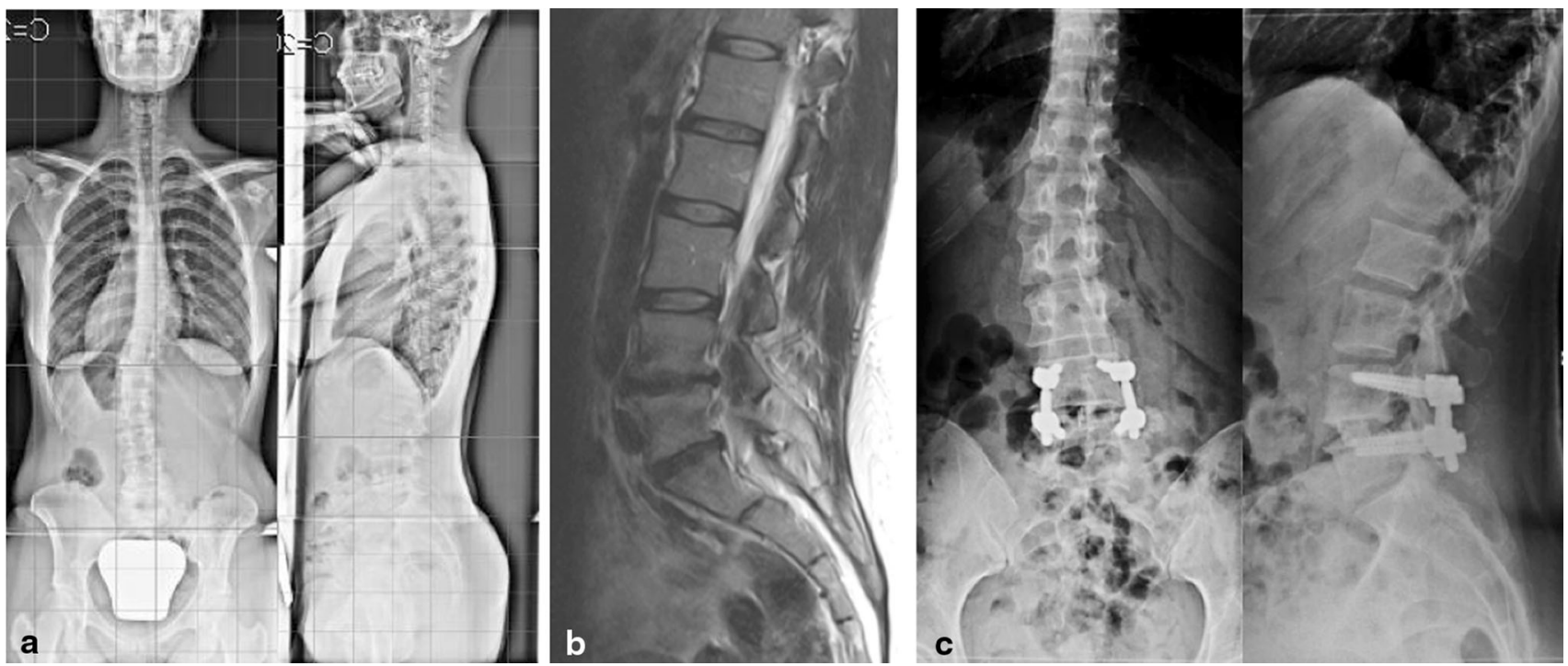

Fig. 1 a-c Type I DSD: limited nonapical symptomatic segment disease at L4-L5, successfully treated with limited fusion to L4-L5

imbalance regardless of the magnitude of sagittal misalignment in standing films, as they could be corrected without the use of aggressive tricolumnar osteotomieswhich is the rationale for classifying a patient as Type IV. Coronal imbalance is defined as lateral displacement of the C7 plumbline more than $4 \mathrm{~cm}$ from the midsacral vertical line [21].

\section{Results}

Four categories of deformity have been identified and described (Table 1):

Type I Limited nonapical segment disease: The diseased (symptomatic) segments do not involve the apical area of the main lumbar/thoracolumbar curve and no significant sagittal imbalance is present. In these cases, and depending on the main symptoms and the anatomy of the lesion, decompression, fusion or both can be limited to the diseased, symptomatic segments. Usually, no correction of the curve has to be planned, as treatment far from the apex of the main curve (decompression if stability is preserved and or fusion when it is not) will not likely compromise the severity of the deformity or cause junctional problems (Fig. 1). In the particular case of a Type I lumbar curve with lower end vertebra at L5 with fusion planned at the L4-L5 level, the lumbosacral junction should be included in the fusion if the L5-S1 segment is significantly degenerated or spondylolysis-spondylolisthesis is present at that level.

Type II Limited apical segment disease: Symptomatic DSD is present at or next to the apex of the main curve. No or minor sagittal imbalance is present $\left(<25^{\circ}\right.$ of loss of lordosis). In these cases (coronal Cobb angle is usually below $25^{\circ}$ ), the spine has to be fused across the apex of the coronal curve to reduce the risk of postoperative junctional disease. If the apex is a vertebra, usually fusion has to be extended one level above and one level below. If the apex is a disc, fusion has to be extended to the two vertebrae above and below the disc (in some early cases, if only the apical disc is degenerated, just one disc can be fused making possible a good curve correction with monosegmental instrumentation). In this category, discs adjacent to the fusion can still be flexible in supine bending films. In such cases, we have observed that correction of the apical area of the curve can provide fair correction of the deformity (Fig. 2) and we hypothesize that correction could slow the progression of the deformity.

Type III Extended (apical and nonapical) segment disease with minor or no sagittal imbalance: Segment degeneration affects both the apical and the end area of the curve. Frequently, coronal Cobb angle is above $25^{\circ}$. In this curve pattern we suggest that fusion be extended to all the symptomatic and degenerated discs, implying frequently fusion from end vertebra to end vertebra (Fig. 3). A particular case of Type III is that of a curve with degenerative changes not only in the main curve, but also affecting the fractional adjacent curves, in most cases the lumbosacral or the thoracolumbar area. If severe disc degeneration is present in the lumbosacral or thoracolumbar junction, the affected adjacent curve should be included in the fusion to reduce the risk of developing a symptomatic junctional disease or deformity. When fusion of the curve is associated with correction of the coronal deformity, the rigidity of the adjacent fractional curves has to be evaluated to avoid coronal decompensation. As 
Fig. 2 a-f Type II DSD. The

apex of the curve is the diseased L3-L4 disc. Segmental sagittal deformity is present, though not still significant for the global alignment. Correction with fusion limited to the apical scoliotic disc restores alignment and alleviates symptoms
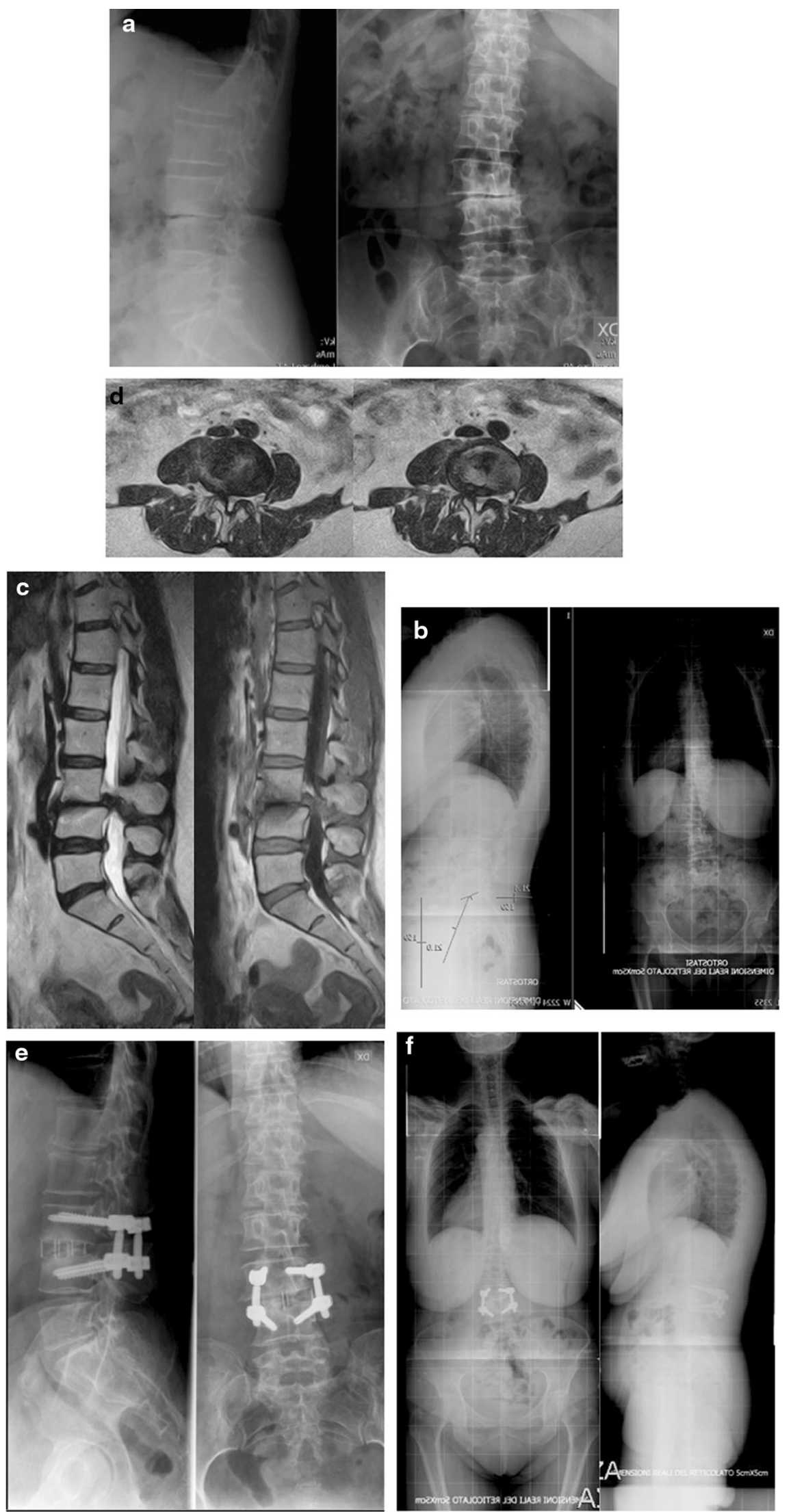


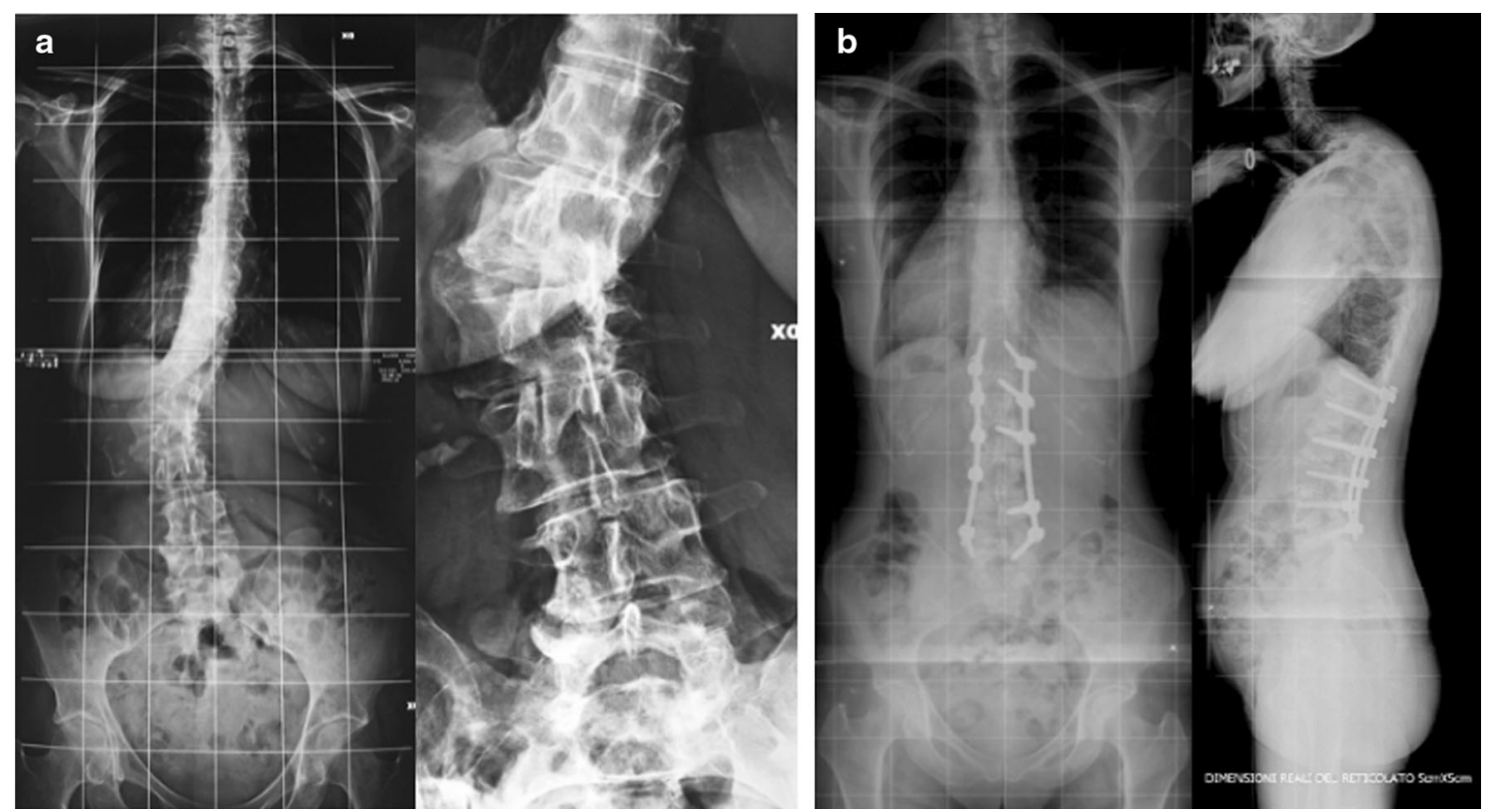

Fig. 3 a, b Type III DSD in adult de novo lumbar scoliosis. After a minimally invasive anterior procedure at T12-L5 and posterior percutaneous fixation, the magnitude of the curve is reduced from $38^{\circ}$ to $2^{\circ}$. Immediate postoperative standing films show good sagittal and coronal profile
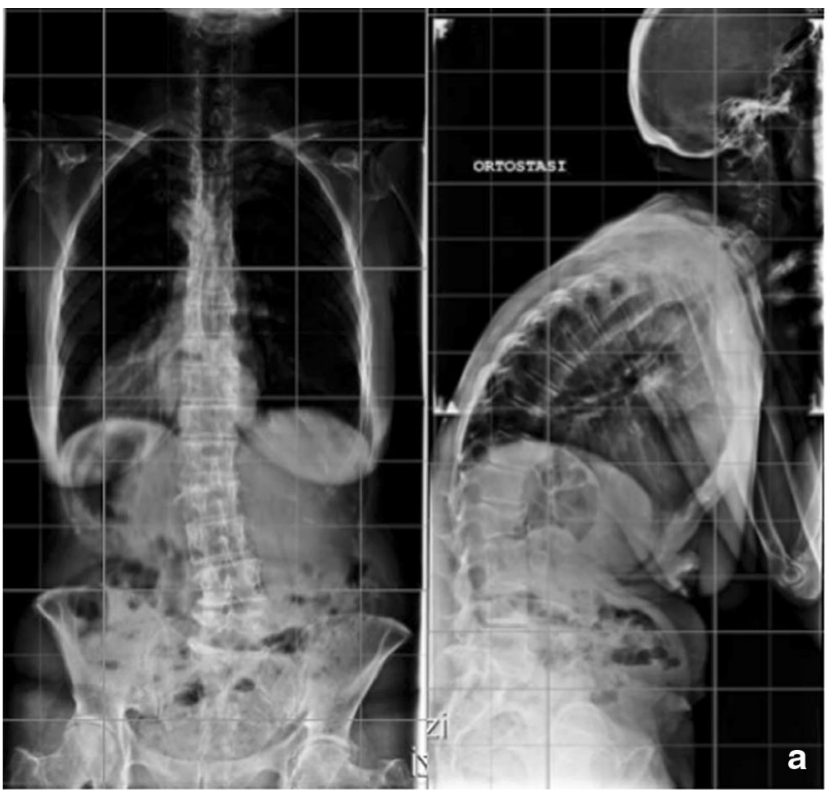

Fig. 4 a-c Type IVa DSD. Severe sagittal imbalance without coronal imbalance. Extensive instrumentation and fusion from T2 to pelvis with pedicle subtraction osteotomy at L4 and Smith-Petersen

a general rule, if correction of the main curve is superior to the flexibility of the adjacent fractional curves in supine side-bending films, these should be included in the fusion to avoid coronal decompensation. Surgical planning should consider the sagittal alignment of the thoracolumbar and lumbosacral areas. Severe thoracolumbar junction kyphosis should be corrected and in such case
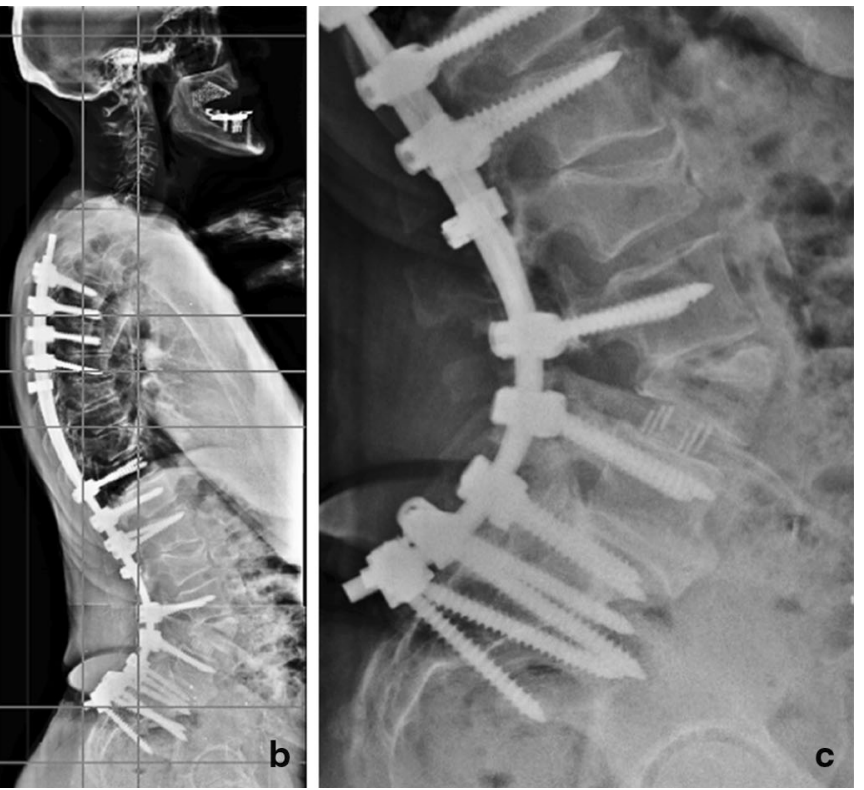

osteotomy at L2-L3 and L3-L4 was needed to provide extra $70^{\circ}$ of lordosis and a stable thoracic spine

fusion should include this area, usually to T10. Severe loss of lordosis of the lumbosacral junction can also be an indication for fusion to the sacrum in a Type III deformity, to improve sagittal alignment.

Type IV Imbalanced spine: Any coronal deformity with severe sagittal imbalance (more than $25^{\circ}$ of sagittal correction are needed) is present and normal sagittal 


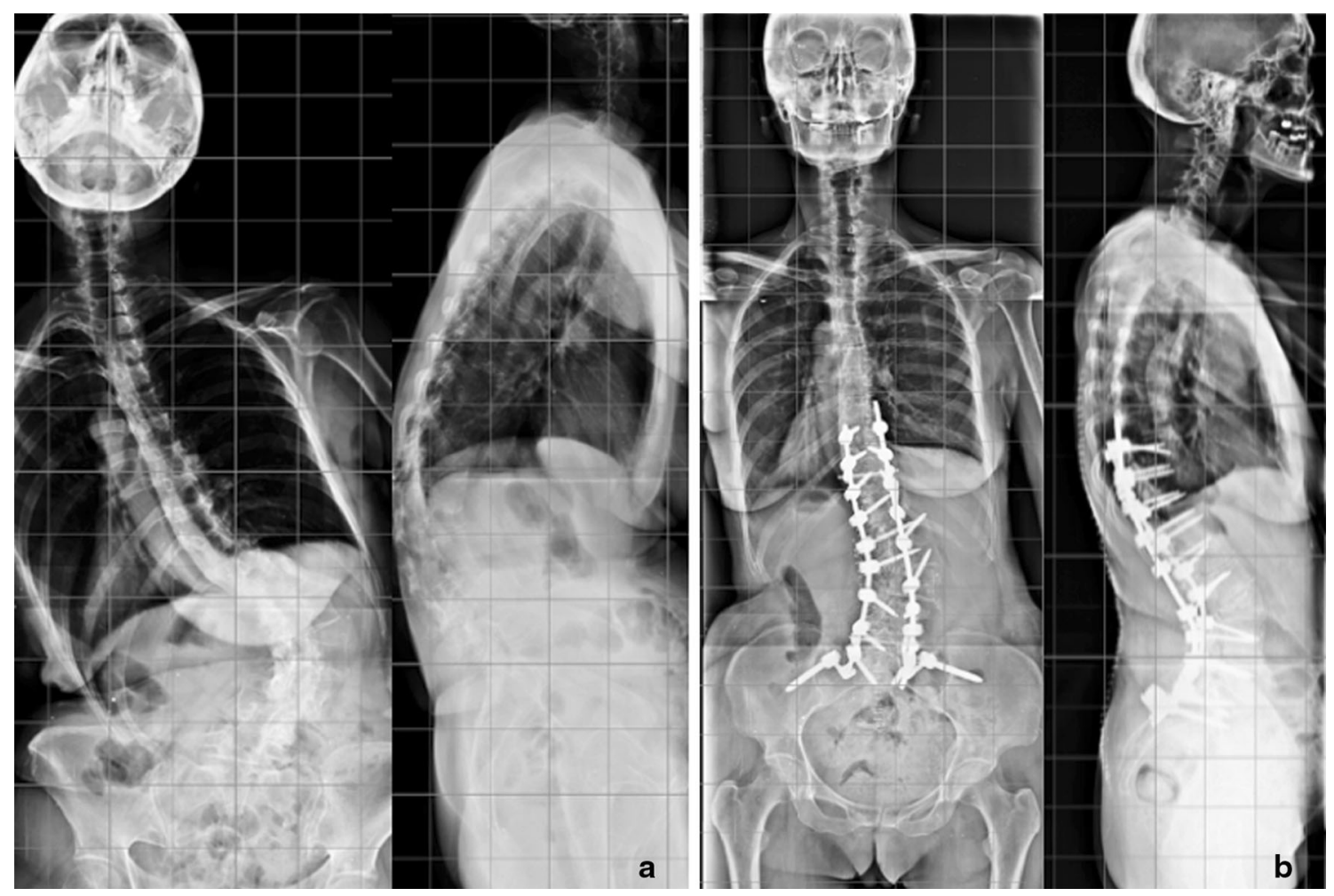

Fig. 5 a, b Type IVb DSD. Severe sagittal and coronal imbalance is present. Release of the rigid area of the sagittal and coronal deformity with asymmetrical PSO at L3 and fusion from T10 to pelvis were needed to correct coronal and sagittal alignment

alignment is not achieved on extension films. This category has two subtypes:

Type IVa Sagittally imbalanced: Coronal imbalance is not present or is $<4 \mathrm{~cm}$ (Fig. 4).

Type IVb Sagittally and coronally imbalanced: In addition to sagittal imbalance, coronal imbalance is more than $4 \mathrm{~cm}$ (Fig. 5).

Type IV deformities cannot reliably be corrected in the sagittal plane by conventional surgical methods, such as instrumentation and fusion without anterior release or osteotomies [21]. At the present moment, the technique most frequently applied in these cases [14] is PSO, which implies a more technically demanding procedure and a higher surgical risk [6]. This justifies including severe imbalance in a different category.

\section{Discussion}

The present classification is based on the position of the symptomatic and degenerated segments with respect to the apical and end area of the main lumbar or thoracolumbar curve and on the (mainly sagittal) imbalance status. One of the key aspects in the clinical application of this classification system is the identification of the symptomatic segments and of the degenerated levels. Symptomatic levels are those motion segments of the spine that cause pain or nerve compression. These levels must be treated to improve patients' symptoms. Furthermore, degenerated, but not symptomatic levels can become symptomatic or cause progression of the deformity if they are left untreated, and for this reason they should be identified and taken into account in the surgical plan.

Unfortunately, there is no exact rule to identify the symptomatic segments of the spine in an adult with deformity. However, some findings are more likely to correlate with symptoms in these patients: laterolisthesis, especially in the lower segments of the lumbar spine, foraminal stenosis, lateral recess or central stenosis and spondylolisthesis when in concordance with the clinical picture of the patient (i.e., concordance between nerve root pain and location of nerve compression or concordance between the area where back pain is perceived and the level of latero- or spondylolisthesis). When present, these give the clinician a good chance to identify the symptomatic area of the spine. Less specific, yet frequently helpful in identifying the symptomatic area of the spine, are Modic changes in the concavity of the coronally asymmetrical discs (specially, Modic I) [22, 23], severe facet joint degeneration or facet joint effusion, and severe disc collapse without ankylosis. Conversely, modest degenerative changes such as slight decrease of disc height, 
low intensity of the disc in MRI (a black disc) or a circumferentially bulging disc without other pathologic changes indicate a lower probability that the segment is symptomatic. Clinicians should (as in the treatment of degenerative disc disease) combine the evaluation of plain radiograph dynamic films, CT and/or MR scans with clinical examination to determine the symptomatic segments. Other diagnostic tools such as facet joint blocks, selective nerve blocks, provocative discography or anaesthetic discography can be useful in selected patients to identify the target motion segments.

Degenerated (yet not symptomatic levels) should be similarly identified. The clinician should evaluate the convenience of including them in the fusion, especially if they are immediately adjacent to the levels to be fused or a long fusion is planned.

Types I and II curves can usually be treated by selective fusion, limited to the degenerated and symptomatic segment or segments. Type II requires extension across the apex of the main coronal curve if only one of the periapical segments were diseased, to reduce the risk of junctional disease in the short term. Practical consequences of the possibility of treating these patterns with selective fusions are reduced surgical risk, the containment of the postoperative limitation of spinal motion and the feasibility of surgical treatment (correction and/or fusion) by minimally invasive fusion techniques in Types I and II patterns.

Type III curves present an extended segment disease, requiring an extensive fusion, generally from end to end vertebra. As these curves have no or slight sagittal misalignment, simpler methods of sagittal correction, such as interbody cage placement with or without SPO, can be sufficient to achieve satisfactory correction. Minimally invasive techniques (yet, with more difficulties) can, in some cases, still be applied with success to this pattern.

Type IV curves have severe balance problems. Most of these curves need aggressive corrective methods, such as PSO to correctly balance the spine. At the present moment, minimally invasive techniques allowing a predictable surgical outcome are not available (though they will probably be described in future). Surgeons and patients should be prepared for extensive surgical procedures with a higher risk of complications.

As shown, this classification can assist in the decision to perform a limited or more extensive surgery. It is also useful to some extent to select the fusion area of the curve. Furthermore, it can be helpful to decide wether a minimally invasive technique (as multilevel transforaminal interbody fusion or lateral lumbar interbody fusion) is enough to provide adequate treatment. This classification allows for interpretation of the deformity pattern and surgical planning, in terms of surgical technique and selection of fusion area. Further research is necessary to validate the reliability of the classification and its usefulness to guide surgical planning.

A good classification should express a gradient of severity. It is intuitive that Type I is a less severe category than Type II, and the same can be said through Types III and IV. Surgical risk and complication rate is likely to correlate fairly well with this classification's categories. Further research is necessary to confirm these hypotheses.

It could be argued that there is no need for a new classification system of adult deformity, as there exists a comprehensive, validated classification as the SRSSchwab's classification [11]. In its current version, the SRS classification of adult deformity defines four types: $\mathrm{T}$ (thoracic only) when only the thoracic curve is more than $30^{\circ}$ in the coronal plane, $\mathrm{L}$ (thoracolumbar or lumbar only) when only the thoracolumbar or lumbar curve is more than $30^{\circ}$ in the coronal plane, D (double curve when both the thoracolumbar/lumbar and the thoracic coronal curves are more than $30^{\circ}$ ) and no major coronal deformity when all coronal curves are $<30^{\circ}$. Additionally, it includes three sagittal modifiers (pelvic incidence minus lumbar lordosis, global sagittal alignment-measured with sagittal translation of C7 plumbline- and pelvic tilt).

The SRS-Schwab's classification has important strengths. It has demonstrated (for the entire grade of curves) an "almost perfect" intrarater agreement and a "substantial" interrater agreement. The three sagittal modifiers demonstrated correlation with clinical outcomes in terms of pain and disability (the latter measured with the Oswestry Disability Index), providing also a clinical validation of the classification method.

Though a major improvement compared to the previous classification systems of adult deformity (it is reproducible, simple to use and correlates with clinical outcomes-i.e., the sagittal modifiers) and some of its assumptions are arbitrary (the thresholds for the $+/++$ subtypes in the three sagittal modifiers, and the threshold of $30^{\circ}$ to distinguish between major and minor curves), the SRS-Schwab's classification's most relevant limitation is that it does not give clear guidelines for treatment (with the important exception of the need for sagittal correction when sagittal modifiers are present). In our opinion, in those adults needing treatment for lumbar/thoracolumbar deformity, two other major concerns are the extension of the fusion area, as both the aggressivity of the surgical procedure and the postoperative functional limitations will strongly depend on it, and the need for severe correction in the sagittal plane by three-columnar osteotomies or similar techniques, as their use is a significant predictor of surgical risk [6]. Another shortcoming of the SRS-Schwab's classification is that it does not clearly differentiate between adolescent and adult idiopathic scoliosis with secondary degeneration and the "true" de novo scoliosis which is 
basically the consequences of a mono- to multilevel disc disease. The de novo scoliosis is more frequent today in an ever-aging society than the so-called idiopathic scoliosis and cannot be addressed with concepts of idiopathic scoliosis surgery. The treatment of true (de novo) degenerated scoliosis has to balance between the necessity of treatment and the involved immediate and late risks, and aggravation related to the local pathomorphology and the general, usually polymorbid condition of the patient. For this reason a system that provides guidance for selective fusion in adult lumbar/thoracolumbar deformity is desirable.

The classification system we propose will hopefully give more help regarding the surgical strategy, both in the "L" type and in the "N" type with mainly lumbar or thoracolumbar involvement of the SRS-Schwab classification. The specific aims of the classification that we propose are to help the surgeon in the selection of the fusion area and to assist in the choice of an adequate strategy to correct sagittal misalignment, if present. Additionally, our proposed classification can be helpful to better analyze and compare subgroups of patients that the SRS-Schwab's classification does not describe in sufficient detail. Finally, our classification bridges the gap between simple degenerative disease of the lumbar and thoracolumbar spine and true deformity.

Though many of the "minor" scoliotic deformities can be considered as simple degenerative disc disease cases, surgical planning has to consider the deformity to avoid undesirable effects as decompensation or junctional problems. In fact, the most frequent adult lumbar and thoracolumbar deformity is degenerative (or "de novo") scoliosis. De novo scoliosis presents as a continuum from minimal deformity due to asymmetrical disc collapse, usually in the mid lumbar spine (as in Type II deformity) to degenerative disc disease all over the main curve (as in Type III) and finally to severe deformity with both sagittal imbalance and coronal (as in Type IV). This spectrum of deformity is not captured by current classification systems that fail to recognize the evolution in severity from asymmetrical disc disease to severe deformity. A classification system that does not include the spectrum from early to late phases of the disease can be of limited usefulness.

The presented classification is a first attempt to structure the decision making for treatment in this complex pathology. The strengths of the proposal are the use of a classification to assist surgical planning, the introduction of a guide to selective fusion and rules to reduce the risk of adjacent segment disease, the inclusion of consideration of the sagittal predictors of outcome and a threshold for considering aggressive methods of correction of the sagittal deformity. This proposal needs to be supported by the evaluation of a sufficient number of cases with their outcomes and evidence from future research that will likely help to refine it.

Conflict of interest None of the authors has any potential conflict of interest.

Open Access This article is distributed under the terms of the Creative Commons Attribution License which permits any use, distribution, and reproduction in any medium, provided the original author(s) and the source are credited.

\section{References}

1. Aebi M (2005) The adult scoliosis. Eur Spine J 14:925-948

2. Ploumis A, Liu H, Mehbod AA (2009) A correlation of radiographic and functional measurements in adult degenerative scoliosis. Spine 34:1581-1584

3. Mac-Thiong JM, Transfeldt EE, Mehbod AA (2009) Can C7 plumbline and gravity line predict health related quality of life in adult scoliosis? Spine 34:e519-e527

4. Glassman SD, Berven S, Bridwell K, Horton W, Dimar JR (2005) Correlation of radiographic parameters and clinical symptoms in adult scoliosis. Spine 30:682-688

5. Smith JS, Shaffrey CI, Glassman SD et al (2011) Risk-benefit assessment of surgery for adult scoliosis an analysis based on patient age. Spine 36:817-824

6. Smith JS, Sansur ChA, Donaldson WF (2011) Short-term morbidity and mortality associated with correction of thoracolumbar fixed sagittal plane deformity. A report from the Scoliosis Research Society Morbidity and Mortality Committee. Spine 36:958-964

7. Transfeldt EE, Topp R, Mehbod AA (2010) Surgical outcomes of decompression, decompression with limited fusion, and decompression with full curve fusion for degenerative scoliosis with radiculopathy. Spine 35:1872-1875

8. Schwab F, el-Fegoun AB, Gamez L, Goodman H, Farcy JP (2005) A lumbar classification of scoliosis in the adult patient: preliminary approach. Spine 30:1670-1673

9. Lowe T, Berven SH, Schwab FJ, Bridwell KH (2006) The SRS classification for adult spinal deformity. Building on the King/ Moe and Lenke classification systems. Spine 31:S119-S125

10. Schwab F, Lafage V, Farcy JP, Bridwell K, Glassman S, Ondra S, Lowe T, Shainline M (2007) Surgical rates and operative outcome analysis in thoracolumbar and lumbar major adult scoliosis: application of the new adult deformity classification. Spine 32:2723-2730

11. Schwab F, Ungar B, Blondel B, Buchowski J, Coe J, Deinlein D, DeWald C, Mehdian H, Shaffrey C, Tribus C, Lafage V (2012) Scoliosis Research Society-Schwab adult spinal deformity classification: a validation study. Spine 37:1077-1082

12. Berjano P, Lamartina C (2013) Far lateral approaches (XLIF) in adult scoliosis. Eur Spine J 22(Suppl 2):S242-S253

13. Schwab F, Patel A, Ungar B, Farcy JP, Lafage V (2010) Adult spinal deformity-postoperative standing imbalance: how much can you tolerate? An overview of key parameters in assessing alignment and planning corrective surgery. Spine 35:2224-2231

14. Bridwell KH (2006) Decision making regarding Smith-Petersen vs. pedicle subtraction osteotomy vs. vertebral column resection for spinal deformity. Spine 31(19 Suppl):S171-S178

15. Vialle R, Levassor N, Rillardon L et al (2005) Radiographic analysis of the sagittal alignment and balance of the spine in asymptomatic subjects. J Bone Jt Surg Am 87:260-267 
16. Rose PS, Bridwell KH, Lenke LG, Cronen GA, Mulconrey DS, Buchowski JM, Kim YJ (2009) Role of pelvic incidence, thoracic kyphosis, and patient factors on sagittal plane correction following pedicle subtraction osteotomy. Spine 34:785-791

17. Berjano P, Cecchinato R, Damilano M, Morselli C, Sansone V, Lamartina C (2013) Preoperative calculation of the necessary correction in sagittal imbalance surgery: validation of three predictive methods. Eur Spine J 22(Suppl 6):S847-S852. doi:10. 1007/s00586-013-3025-9

18. Lamartina C, Berjano P, Petruzzi M, Sinigaglia A, Casero G, Cecchinato R, Damilano M, Bassani R (2012) Criteria to restore the sagittal balance in deformity and degenerative spondylolisthesis. Eur Spine J 21(Suppl 1):S27-S31. doi:10.1007/s00586012-2236-9

19. Aurouer N, Obeid I, Gille O, Pointillart V, Vital JM (2009) Computerized preoperative planning for correction of sagittal deformity of the spine. Surg Radiol Anat 31:781-792
20. Le Huec JC, Leijssen P, Duarte M, Aunoble S (2011) Thoracolumbar imbalance analysis for osteotomy planification using a new method: FBI technique. Eur Spine J 20:669-680

21. Berjano P, Lamartina C (2013) Answer to the Letter to the Editor of T.A. Mattei concerning "Far lateral approaches (XLIF) in adult scoliosis" by P. Berjano and C. Lamartina (Eur Spine J. 2012 Jul 27. [Epub ahead of print]). Eur Spine J 22(5): 1186-1190. doi:10.1007/s00586-012-2637-9

22. Esposito P, Pinheiro-Franco JL, Froelich S, Maitrot D (2006) Predictive value of MRI vertebral end-plate signal changes (Modic) on outcome of surgically treated degenerative disc disease. Results of a cohort study including 60 patients. Neurochirurgie 52(4):315-322

23. Braithwaite I, White J, Saifuddin A, Renton P, Taylor BA (1998) Vertebral end-plate (Modic) changes on lumbar spine MRI: correlation with pain reproduction at lumbar discography. Eur Spine J 7(5):363-368 